\title{
User Centred Design in a Learning Environment Based on a Computer Algebra System
}

\author{
Dominique Lenne and Jean-Michel Gélis \\ Institut National de Recherche Pédagogique, 91, rue Gabriel Péri, Montrouge, France \\ dominique.lenne@inrp.fr
}

Keywords: user interface, didactics, open learning systems, integration of ICT, problem solving

\begin{abstract}
Though they have not been designed for this usage, computer algebra systems are increasingly used in learning situations. In order to improve their didactic capabilities we study the possibility to graft mathematics interactive learning environments onto them. We present in this paper our design approach that relies on Human Computer Interaction methods and integrates mathematics learning knowledge. This approach has been applied to design a learning environment, called Limites, that helps students to study, work out and justify limits of rational functions. We present the main features of this environment, focusing on three points: algebraic expressions manipulation, conjectures and proofs.
\end{abstract}

\section{INTRODUCTION}

Though they have not been designed for this usage, professional software packages are often used in learning and training situations (Cheikes et al. 1998). That is the case with Computer Algebra Systems (CAS), such as Maple, Derive or Mathematica, which are increasingly used in mathematics teaching and learning.

Students are special users of these software packages because their goal in using them is not only to solve a problem but also to improve their knowledge. Unlike professional users, they often have a very partial, but strongly evolving knowledge of the domain covered by the software. 
Moreover, professional tools are generally complex and the time needed to master them is often too long in comparison with the short time during which students will have to use them.

Taking into account these problems, we study the possibility to graft (Pachet et al. 1994) learning environments onto those tools. To design these environments we adopt user centred methods inspired of the Human Computer Interaction domain (Norman 1986), considering two types of users: students and teachers.

In this paper, we describe our approach and we show how it has been applied to Limites, a learning environment based on the Maple computer algebra system and centred on problem solving in the limits domain. This environment provides students with tools to study, work out and justify limits of numerical functions and provides teachers with tools to parameter and to adapt it.

\section{CAS-BASED ENVIRONMENTS}

Much research work have been done about the integration of CAS in mathematics education. In the eighties, artificial intelligence research tried to integrate them in problem solving systems and to construct explanations using solutions produced by these systems (Vivet 1984). In the nineties, when CAS became usable on microcomputers and on handheld calculators, mathematics education researchers (Artigue and Lagrange 1998, Trouche 1996) analysed the conditions of their integration in education. They showed that this integration was not as easy as one could firstly think and stressed the importance of the learning situation. Following cognitive ergonomics works (Rabardel 1995), they particularly focused on the instrumentation process and on the identification of students action schemes.

Our approach consists in designing, on the top of a CAS, environments that facilitate learning in specific mathematical domains, and reduce the complexity of action schemes observed in existing CAS.

\section{USER CENTERED DESIGN}

We present below some key points of our approach that relies on one hand on a study of CAS utility and usability in mathematics learning and on the other hand on an analysis of students problem solving activity. It takes into account didactic goals and allows teachers to tailor the environment to their needs. 


\subsection{Utility and usability of CAS in mathematics learning}

Computer Algebra Systems are powerful that can make most of the calculations (numeric or symbolic) needed at different levels (secondary schools or university). Moreover they have extended capacities in graphical representation and animation.

However, the integration of CAS in mathematics' teaching raises many questions (Lagrange 2000), particularly relating to the relationship between conceptual and technical aspects. For the most part, the existing CAS have been developed for research and industry and not for learning. Above all designed in order to be efficient, they use complex algorithms that do not match the methods taught to the students. They do not follow the principle of 'cognitive fidelity' (Beeson 1998, Nicaud et al. 1999) needed in a learning environment.

CAS have many flaws regarding their interface and help capabilities (Lenne and Gélis 1998).

- The great number of functions they propose (more than 2700 for Maple)

- The unsuited power of some functions (automatic simplifications, integration of several calculus steps in one function)

- Conversely, the impossibility to perform other simple functions (unneeded in a powerful software)

- The difficulty to take into account definition domains

- The resolution of equations in the complex numbers set, which leads to results difficult to understand for some students

- Many ergonomic flaws such as the difficulty to visualise the state of variables and functions and to spread a modification.

Some of these flaws lead to usability problems related to the interface, others to utility problems related to the functional kernel that are more difficult to deal with.

Note that most of these flaws are seen, conversely, as advantages in professional situations.

\subsection{Students' activity analysis}

In order to conceive a learning environment in the limits domain, we analysed students' activity relying on their paper and pencil work and on their work with the aid of a classic CAS. Their foreseeable future activity in the learning environment is based on this analysis, but it is also guided by learning goals defined by the teachers and mathematics' teaching specialists involved in our project. 


\subsubsection{Paper and pencil activity}

In a paper-and-pencil context, working on limits implies carrying out two different tasks. The first one consists in applying theorems and properties in order to calculate the limit. The second one allows applying some transformations when the limit evaluation leads to an indeterminate form (such as $0 \times \infty$ or $+\infty-\infty$ ).

Teachers note many difficulties when students carry out these tasks, such as misapplication of theorems or calculus errors when transforming expressions. Moreover, students often manage to fit in both of these tasks, which leads to some confusion. In Limites, the learning environment we designed, these two tasks have been clearly separated, and the calculations are made by CAS.

\subsubsection{CAS-aided problem solving activity}

With a classical CAS, the student's task consists in finding a sequence of commands or functions leading to a 'good' expression, i.e. an expression that can be used to calculate the limit (if it exists). When executing this task, students encounter the above mentioned CAS utilisation problems. Among them, the major problem is that available functions are often too powerful because they have not been designed to facilitate learning but in order to be efficient.

Moreover, a CAS provides calculus functions but it is helpless in producing proofs. For example, the value of a limit can be calculated but there are no means (e.g. reference limits, theorems) to prove it.

\subsection{Didactic goals}

To experiment with this approach, we chose the domain of numerical functions' limits at a high school level. This domain is interesting from several viewpoints: it implies to work on algebraic expressions and on their syntactical structure, it implies the use of theorems and reference limits to prove a result and it allows one to tackle the analysis domain by using inequalities to evaluate some particular limits. Moreover conjectures can be obtained by numerical or graphical means.

\subsubsection{Learning the limit concept}

To identify the difficulties encountered by high school students in limits learning, we carried out a preliminary pilot study. Students had to solve 
limits problems in a paper-and-pencil mode. The observed difficulties concerned the three following main points.

- Manipulation of expressions: many difficulties appear when students try to apply transformations on expressions. They sometimes have difficulties in identifying sub-expressions and they often choose complicated or inappropriate transformations in order to obtain an expression with which the limit can be calculated; moreover they make many calculation errors in applying them.

- Proof: students often have many difficulties in justifying their results. They use incorrect operations involving $+\infty$ or $-\infty$, abusively generalise some theorems (for example, they use the limit of the higher degree term for a non-polynomial expression) and often have little knowledge on reference limits.

- Conjectures: when the limit does not exist or when it is uneasy to calculate, students do not always think to make conjectures by using numerical or graphical means. Moreover, they do not always know the difference between a conjecture and a proof.

\subsubsection{The learning situation}

The learning situation we propose in the Limites environment aims at taking into account these difficulties. It puts the emphasis on the students activity by providing them with relevant calculus, conjecture and proof tools.

Furthermore, we aim at encouraging links between numeric, graphic and symbolic registers, in order to facilitate conjectures (Artigue and Lagrange 1998), specially in situations where students are stuck.

Lastly, didactic goals change while students knowledge evolves. For example, when the limit concept has just been introduced, students only know elementary theorems and few reference limits. They have to justify each step of reasoning. When the notion begins to be mastered, they can use more powerful theorems and do not have to justify all the elementary steps of reasoning.

\section{$3.4 \quad$ Teachers' viewpoint}

The learning situations we consider involve teachers. Therefore, we have to analyse their tasks and to take into account their needs. We note other authors (Van Labeke et al. 1999) that have stressed the importance of integrating teachers in the design process.

In our project a key issue has been to give teachers means to tailor the environment to the students level and to the kind of justifications they 
expect. As a pilot study we undertook has shown, the expected justifications vary not only according to the teaching level but also to the teacher himself.

\section{THE LIMITES LEARNING ENVIRONMENT}

The Limites learning environment has been designed in accordance to the above didactic goals and in order to reduce difficulties that students encounter both in paper-and-pencil and in CAS context. We describe below its architecture and its main features, focusing on three points: algebraic expressions' manipulation, conjectures and proofs.

\subsection{Architecture}

Limites is composed of two main units, the first one taking in charge the interaction with the student and the second one the adaptation of its requests to the CAS (see Figure 1). These two units have been written in Delphi (Pascal Object) and the CAS we used is Maple. Relating to the previous section, the adaptation unit takes charge of CAS utility problems while the interaction unit takes charge of usability problems.

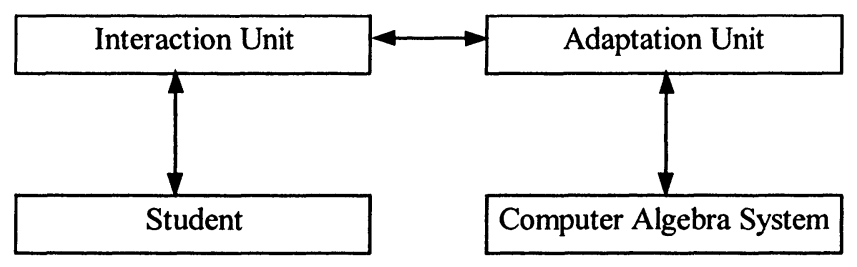

Figure 1. Architecture of Limites

In addition, the adaptation unit makes the interaction unit independent from the CAS by including a representation of its expected capacities.

\subsection{Manipulation of expressions}

Manipulation of expressions can be divided into at least three tasks: entering an expression, selecting a sub-expression and applying a transformation. 


\subsubsection{Entering an expression}

In the current version of the environment, students have to enter an expression under its linear form. It is then parsed and displayed in its twodimensional form. Starting from this representation, expressions or subexpressions can directly be selected in order to calculate a limit or to apply a transformation.

We plan to add later a mixed expression entry mode, allowing the student to use both of these representations. Both of them can indeed be useful to lead to a better understanding of expressions structures.

\subsubsection{Selecting a sub-expression}

In order to transform an expression, students have to recognise and to work on its sub-expressions. This task is sometimes difficult for students in paper and pencil mode and it is even more complicated with a CAS. Limites helps students in this task by allowing them to visualise the expression or sub-expression they can select. When moving the mouse over the expression, different moving frames are drawn, showing sub-expressions that can be selected by clicking (Figure 2).

$$
\frac{x^{2}+x+1}{x^{2}-3 x+2} \quad \frac{x^{2}+x+1}{x^{2}-3 x+2} \quad \frac{x^{2}+x+1}{x^{2}-3 x+2} \frac{x^{2}+x+1}{x^{2}-3 x+2}
$$

Figure 2. Visualisation of sub-expressions

\subsubsection{Applying transformations}

In order to calculate limits, students often have to perform transformations (e.g. expand, factor, simplify), particularly when the current expression leads to an indeterminate limit. In Limites, to achieve this task, they have (1) to select a sub-expression (2) to choose a transformation in a list. Some transformations are always available in the list, others vary according to the expression type. The chosen transformation is applied to the expression by the system.

For example, to study the limit of $x^{2}-3 x+2$ when $\mathrm{x}$ tends towards infinity, students who do not know that the limit of a polynomial, function when $x$ tends towards infinity, is the same as its higher degree term limit have to 
solve the non-determination by using the 'factor' transformation leading to $x^{2}\left(1-3 / x+2 / x^{2}\right)$.

\subsection{Conjectures}

Students have to be able to make conjectures either on the value of a limit or on its non-existence (e.g. for some trigonometric functions when the variable tends towards infinity). Numerical and graphical tools can help them in Limites. They look like other tools in classical CAS, but in addition, non-linear scales that can give a better but unusual insight of the behaviour of a function are provided.

Conjecture tools are available at the same level as proof tools and a clear difference is made between these two types of tools.

\subsection{Proof}

The limit concept is studied at different teaching levels. Teachers justification requirements vary depending on the teaching level. At an early stage, each step has to be justified, while later some evident results can be omitted. An important feature of a learning environment is therefore to provide several levels.

By now, we have implemented three levels (these levels can easily be adapted and more levels can be considered).

$$
\begin{aligned}
& \lim _{x \rightarrow+\infty} 1=1 \quad \text { Limite de référence } \\
& \lim _{x \rightarrow+\infty} 3=3 \quad \text { Limite de référence } \\
& \lim _{x \rightarrow+\infty} x^{2}=+\infty \quad \text { Limite de référence } \\
& \lim _{x \rightarrow+\infty} \frac{3}{x^{2}}=0 \quad \text { Quotient }
\end{aligned}
$$

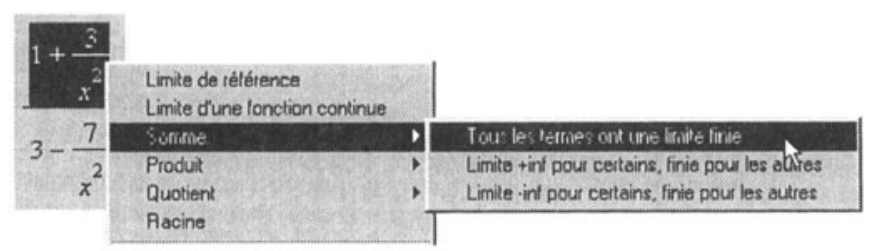

Figure 3. A limit calculus at the first level 
At the first level (Figure 3), the proof has to be stated step by step with elementary theorems. At the upper levels, some justifications can be implicitly admitted and more powerful theorems become available. In our example, at the second level the number of justification steps needed is reduced to two. At the third level, a new theorem relating to the limit of a polynomial function when the variable tends towards infinity allows the student to perform the calculus in only one step.

\section{CONCLUSION AND FUTURE WORK}

In this paper, we presented the learning environment Limites which is based on a CAS. We showed how the design process has been centred on the users of this environment. On one hand students tasks and activities with and without the CAS have been precisely analysed. On the other hand teachers' needs and requirements and didactic goals have been taken into account. The expected outcome is a better integration of this environment in classroom situations.

A prototype of this environment is fully functional and could be used as is in high school classrooms. We are evaluating this prototype with students and teachers, both from a pedagogical and from a HCI point of view. The first results showed the great ease of use of the environment. We now try to observe the mathematical work of students with the environment and to see to what extent it helps them in constructing the limit concept.

Our future work will concern the extension of the mathematical domain by allowing students to calculate limits thanks to relevant inequalities.

\section{REFERENCES}

Artigue, M. and Lagrange, J. B. (1998) Instrumentation et écologie didactique de calculatrices complexes: éléments d'analyse à partir d'expérimentations en classe de première $\mathrm{S}$. Actes du colloque "Calculatices symboliques et géométriques dans l'enseignement des mathématiques", IREM de Montpellier, pp. 15-38.

Beeson, M. (1998) Design Principles of Mathpert: software to support education in algebra and calculus. In Computer Human Interaction in Scientific Computation, N. Kajler (ed.), Springer-Verlag, pp. 89-114.

Cheikes, B., Geier, M., Hyland, R., Linton F., Rodi, L. and Schaefer, H. P. (1998) Embedded Training for Complex Information Systems. ITS'98, $4^{\text {th }}$ International Conference on Intelligent Tutoring Systems, San Antonio, TX, Springer, pp. 36-45.

Lagrange, J. B. (2000) Approches didactique et cognitive d'un instrument technologique dans l'enseignement Le cas du calcul formel en lycée. Mémoire d'Habilitation à Diriger les Recherches. Université Paris VII. 
Lenne, D. and Gélis, J. M. (1998) Integration of Learning Capabilities into a CAS: the SUITES Environment Example. In Proceedings of 4th International Conference On Computer Aided Learning and Instruction In Science and Engineering, C. Alvegard (ed), Chalmers University, Göteborg, pp. 342-349.

Nicaud, J. F., Bouhineau, D., Varlet, C. and Nguyen-Xuan, A. (1999) Towards a product for teaching formal algebra. In Proceedings of Artificial Intelligence in Education, S. P. Lajoie and M. Vivet (eds.), IOS Press, pp. 207-214.

Norman, D. (1986) User Centered System Design. Lawrence Erlbaum Associates.

Pachet, F., Giroux, S. and Paquette, G. (1994) Pluggable Advisors as Epiphyte Systems. In Proceedings of CALISCE'94, International Conference On Computer Aided Learning and Instruction In Science and Engineering, Telecom, Paris.

Rabardel, P. (1995) Les hommes et les technologies: une approche cognitive des instruments contemporains. Paris, A. Colin.

Trouche, L. (1996) Etude des rapports entre processus de conceptualisation et processus d'instrumentation. Thèse de doctorat, Université de Montpellier.

Van Labeke, N., Aiken, R., Morinet-Lambert, J. and Grandbastien, M. (1999) IF "What is the core of AI \& Education ?" is the Question THEN "Teaching Knowledge" is the Answer. In Artificial Intelligence in Education, S. P. Lajoie and M. Vivet (eds.), IOS Press, pp. 241248.

Vivet, M. (1984) Expertise mathématique et informatique: CAMELIA, un logiciel pour raisonner et calculer. Thèse d'état, Université Paris VI.

\section{BIOGRAPHY}

Dominique Lenne is in charge of research in the 'New Technologies and Education' (TECNE) department of the French institute of pedagogical research (INRP). His current research interests are centred on the design and evaluation of computer algebra learning environments.

Jean-Michel Gélis is senior lecturer at the teacher training institute of Versailles. The work reported in this paper has been carried out with helpful contribution of Hervé Hamon and Michel Enjalbert, qualified high-school mathematics teachers. 